\title{
Melanoma in the Older Patient: Measuring Frailty as an Index of Survival
}

\author{
Julie R. Lange, MD ScM${ }^{1}$, Sewon Kang, $\mathrm{MD}^{2}$, and Charles M. Balch, MD ${ }^{1}$ \\ ${ }^{1}$ Department of Surgery, Johns Hopkins Medicine, Baltimore, MD; ${ }^{2}$ Department of Dermatology, Johns Hopkins \\ Medicine, Baltimore, MD
}

The population continues to age, and with that aging will come increasing numbers of cancer cases diagnosed at older ages, including cutaneous melanoma. The number of people in the United States aged 65 and above is expected to increase from 35 million in 2000 to 86.7 million by $2050 .{ }^{1}$ Age-adjusted mortality rates for all cancers in the United States are higher for patients age 65 and above than for patients younger than 65. For melanoma, both the incidence and mortality rates are higher among older patients. ${ }^{2}$ The explanation for the difference in melanoma outcomes in older patients is likely to be multifactorial; it could be related to differences in biology of cancer itself in older patients; differences in patient physiology, with older patients more likely to have immune system dysfunction or multiple comorbidities; or differences in access to and application of health care resources. ${ }^{3}$ Care of the older melanoma patient presents many challenges, related both to different biology of cancer in older persons and to the different biology of older persons themselves.

The risk of being diagnosed with melanoma increases with age. ${ }^{2}$ In the United States, men between the ages of 40 and 59 years have a 1 in 157 probability of being diagnosed with melanoma, compared to a probability of 1 in 54 at age 70 and older. For women, the probability of being diagnosed with melanoma is 1 in 181 between 40 and 59 years, and increases to 1 in 123 at 70 years and older. ${ }^{4}$ Moreover, older melanoma patients on average possess poorer prognostic factors at diagnosis. Older patients are more likely to have primary melanomas that are thicker, nodular, ulcerated, and located on the head and neck. More new melanoma cases are now diagnosed in people over age

(C) Society of Surgical Oncology 2011

Published Online: 31 August 2011

J. R. Lange, MD ScM

e-mail: jlange@jhmi.edu
60 and with the aging of the population, this trend is expected to continue, with even greater numbers of melanoma cases being diagnosed in older persons in the coming decades. Improvement in the evaluation and treatment of melanoma in the older person will assume increasing importance.

Older patients are not only more likely to be diagnosed with melanoma, but are also more likely to die from this malignancy. In a multivariable analysis of over 17,600 patients, age was an independent poor prognostic factor in patients with stage I-III melanoma. ${ }^{5}$ Age is a persistently significant poor prognostic factor among node-positive melanoma patients. In a recent multivariable analysis of 2313 node-positive melanoma patients from the American Joint Commission on Cancer (AJCC) database, patients age $\geq 70$ years had significantly poorer survival than did younger patients. ${ }^{6}$ The reason for poorer outcomes in older patients remains obscure. It has been suggested that decreased host immunologic function may play a role, although the mechanism of this possibility is not well understood. Other possible explanations for poorer survival among older melanoma patients include more aggressive tumor biology (including increased incidence of acral lentiginous and desmoplastic melanomas), different mechanisms or ease of metastatic spread (including lymphatic dysfunction), limited physiologic reserve, poorer access to care, and under treatment by physicians. ${ }^{7,8}$

Frailty in the elderly is associated with several adverse outcomes including increased risk of hospitalization, falls, impaired mobility, and disability. Frailty develops slowly and has many components including weight loss, weakness, poor endurance, slowness, and low physical activity. Muscle mass peaks in approximately the third decade and declines notably after the sixth decade; thus, sarcopenia is closely associated with increasing age. Sarcopenia can occur as a component of the physiologic changes of aging 
and can be distinct from wasting (from inadequate caloric intake) and from cachexia (loss of lean body mass from illness). ${ }^{9}$ Sarcopenia is sometimes quantified from the appearance of muscle on cross-sectional imaging. In this issue of the Annals of Surgical Oncology, Sabel et al. report on sarcopenia as a prognostic factor in patients with nodepositive melanoma. ${ }^{10}$ In this retrospective study sarcopenia was measured from the psoas muscle size and density on staging computed tomographic scans. This study found that in multivariable analyses, sarcopenia was a better prognostic factor than age; in fact, when sarcopenia was added to the model, age was no longer a statistically significant prognostic factor.

Sarcopenia has been reported previously to be associated with increased risk of cancer recurrence and death, so the reported association of sarcopenia and poorer melanoma prognosis is interesting. ${ }^{11}$ The finding may reflect one of the complex and multiple underlying mechanisms responsible for poorer outcomes in older melanoma patients. Sarcopenia could well be a measurable factor associated with outcomes, but a mechanistic explanation for the association is unknown. Could sarcopenia as a measure of frailty be a useful prognostic factor for melanoma patients? At this point it is unclear, but the issue warrants further investigation, especially in view of the cost of obtaining the information by computed tomographic scans. Sarcopenia as one measure of frailty could be viewed as having value in risk stratification and could offer useful information in clinical decision making, especially in older patients who are being considered for major melanoma surgery or other therapies. Perhaps a more intriguing question is whether sarcopenia could be viewed as a modifiable prognostic factor rather than a mere measure of an underlying physiologic change that results in poorer cancer survival. Further studies to elucidate the mechanisms responsible for poor outcomes in older melanoma patients and to better understand the interactions of the tumor and the host remain important for increasing numbers of older melanoma patients.

\section{REFERENCES}

1. He W, Sengupta M, Velkoff VA, DeBarros, KA. $65+$ in the United States. U.S. Census Bureau. 2005. http://www.census.gov/ prod/2006pubs/p23-209.pdf.

2. Linos E, Swetter SM, Cockburn MG, Colditz GA, Clarke CA. Increasing burden of melanoma in the United States. J Invest Dermatol. 2009;129:1666-74.

3. Lasithiotakis KG, Petrakis IE, Garbe C. Cutaneous melanoma in the elderly: epidemiology, prognosis and treatment. Melanoma Res. 2010;20:163-70.

4. Siegel R, Ward E, Brawley O, Jemal A. Cancer statistics, 2011: the impact of eliminating socioeconomic and racial disparities on premature cancer deaths. CA Cancer J Clin. 2011;61:212-36.

5. Balch CM, Soong SJ, Gershenwald JE, et al. Prognostic factors analysis of 17,600 melanoma patients: validation of the American joint committee on cancer melanoma staging system. J Clin Oncol. 2001;19:3622-34.

6. Balch CM, Gershenwald JE, Soong SJ, et al. Multivariate analysis of prognostic factors among 2,313 patients with stage III melanoma: comparison of nodal micrometastases versus macrometastases. J Clin Oncol. 2010;28:2452-9.

7. Varani J, Warner RL, Gharaee-Kermani M, et al. Vitamin A antagonizes decreased cell growth and elevated collagendegrading matrix metalloproteinases and stimulates collagen accumulation in naturally aged human skin. $J$ Invest Dermatol. 2000;114:480-6.

8. Conway CW, Faries MB, Nicholl MB, et al. Age-related lymphatic dysfunction in melanoma patients. Ann Surg Oncol. 2009;16:1548-52.

9. Pal SK, Katheria V, Hurria A. Evaluating the older patient with cancer: understanding frailty and the geriatric assessment. $C A$ Cancer J Clin. 2010;60:120-32.

10. Sabel MS, Lee J, Cai S, et al. Sarcopenia as a prognostic factor among patients with stage III melanoma. Ann Surg Oncol. 2011. doi:10.1245/s10434-011-1976-9.

11. Prado CM, Lieffers JR, McCargar LJ, et al. Prevalence and clinical implications of sarcopenic obesity in patients with solid tumours of the respiratory and gastrointestinal tracts: a population-based study. Lancet Oncol. 2008;9:629-35. 